\title{
“Ora sí que quién sabe”: Sobre las funciones de ahora sí que como marcador discursivo
}

\author{
Josaphat Enrique Guillén Escamilla - Escuela Nacional de Antropología e Historia \\ josaphat_guillen@enah.edu.mx
}

Rebut / Received: 05/02/20

Acceptat / Accepted: 22/04/20

Resumen. Ora sí que quién sabe. Sobre las funciones de abora sí que como marcador discursivo. El objetivo de este trabajo es caracterizar las funciones pragmáticas que desempeńa ahora si quelora si que como marcador discursivo. Para tal fin, partimos de la propuesta teórica del grupo Val.Es.Co. (Briz, 1993; Pons, 2001) y del análisis de las 108 entrevistas del Corpus Sociolingüístico de la Ciudad de México (Martín Butragueño \& Lastra, 2011, 2012, 2015). A partir del análisis, se pudieron distinguir tres funciones: (i) marca de pertinencia, (ii) ejemplificador y (iii) reformulador. Además, se describieron dos valores secundarios que pueden acompañar al marcador: (a) formulación y (b) modalización. Las tres funciones y el valor secundario de formulación corresponden a los marcadores metadiscursivos, relacionados con la técnica que emplean los hablantes para formular y producir sus mensajes. Mientras que el segundo valor secundario corresponde a los modalizadores, relacionados con la forma en que el hablante se enfrenta al enunciado y la actitud que tiene frente a él.

Palabras clave: pragmática, discurso, marcadores discursivos.

Abstract. Ora si que quién sabe. On functions of abora sí que as discourse marker. Our aim is characterizing the pragmatic functions of ahora si quelorasi que when works as discourse marker. For that end, we start from theoretical framework proposed by Grupo Val.Es.Co. (Briz, 1993; Pons, 2001) and we analyze all 108 interviews of Corpus Sociolingüístico de la Ciudad de México (Martín Butragueño \& Lastra, 2011, 2012, 2015). The analysis shows that ahora si quelorasi que can carry out three functions: (i) mark of pertinency, (ii) exemplifier, and (iii) reformulator. Further, it has been described two secondary values that can accompany to discourse marker: (a) formulation and (b) modalization. The first three functions and secondary value of formulation are compatible with metadiscursive markers that help to speaker to formulate and produce his/ 
her messages. Meanwhile, secondary value of modalization is compatible with modalizers, related with the way in speaker thinks about his/her own utterance, and with the attitude that he/she assume about it.

Keywords: pragmatics, discourse, discourse markers.

\section{Introducción}

El análisis de los marcadores discursivos ha ganado cada vez mayor atención. Actualmente, contamos con descripciones bastante detalladas de varios de ellos; sin embargo, aún existen algunos que han sido menos estudiados, ya sea porque son incipientes o, en cambio, por ser específicos de una variante del español. Un ejemplo de esto último es el marcador ahora si que - y su forma reducida ora si que-, que se ha mostrado como característico de la variante del español de México y cuyo estudio solo ha sido abordado previamente por Aldama y Reig (2016). Nos referimos a casos como:

(1) 106 I: los autobuses por la carretera/ ya no te levantaban/ ya que estaba oscuro// y varias veces pues < pus> estuve a punto de quedarme por ahí/ ¿no?/ en lugares/ totalmente/ alejados de la humanidad (risa)// y yo decía/ “¡ay!” ahora < ora> si que como la llorona/ “¡ay! mis hijos”/ ¿̨no?/ (Entrevista 22). ${ }^{1}$

Al respecto, a pesar de no estar en sus objetivos ofrecer un análisis pragmático detallado, Aldama y Reig (2016) señalan que este marcador cumple con una función de reformulación "que permite al hablante ofrecer una nueva formulación de aquello que acaba de decirse, para corregirlo, explicarlo, modificar sus elecciones léxicas, etc." (p. 25). En otros casos, no hay un primer miembro que reformular, por lo que "solo indica aquello que introduce como el miembro del discurso que ha de tenerse en cuenta para la prosecución del discurso" (p. 26). De tal forma, concluyen que su empleo está asociado con la función más general de formulación (Portolés, 1998), aquella que permite presentar un miembro del discurso "como aquel que transmite satisfactoriamente la intención comunicativa del hablante" (p. 25).

En cuanto a su variación sociolingüística, mencionan que se ha vuelto más común en la variedad del español de la Ciudad de México y, en menor medida, en la de la ciudad de Monterrey. También describen que los hablantes jóvenes favorecen su uso,

1. Los ejemplos pertenecen al Corpus Sociolingüístico de la Ciudad de México (Martín Butragueño \& Lastra, 2011, 2012, 2015). Para su inclusión en el texto se decidió conservar la convención original de etiquetado PRESEEA (2008) (ver Apéndice). 
mientras que los mayores son quienes menos lo utilizan. Asimismo, el grupo de nivel de instrucción alto es el que menos prefiere su empleo, en tanto que el de instrucción media muestra el patrón contrario. En resumen, concluyen que su uso es "una innovación que no goza de gran prestigio en la comunidad, pues ni los hablantes de instrucción alta, ni las mujeres, que como se sabe suelen ser favorecedoras de las variantes de prestigio en su comunidad, favorecen ahora si (que)" (p. 43).

No obstante, este estudio considera como formas análogas ahora si y ahora si que, mientras que aquí defendemos que no se trata del mismo marcador ni el primero es una forma abreviada del segundo, principalmente porque no pueden intercambiarse en los mismos contextos discursivos:

(2) y yo decía/ "iay!” ahora sí, como la llorona/ “ay! mis hijos”

(3) y yo decía/ "jay!" ahora sí que como la llorona/ "jay! mis hijos"

En (2) ahora sí tiene un valor temporal, ya que puede ser parafraseado como esta vez sí, mientras que en (3) ahora si que cuenta con un valor de ilustración o ejemplificación. Así, la diferencia está en el ámbito en el que ejercen su influencia; ahora sí lo hace sobre la situación de la enunciación, especificando aspectos temporales, en tanto que ahora sí que lo hace sobre lo enunciado, reflejando la actitud del hablante.

Así pues, nuestro objetivo es caracterizar las funciones que puede desempeñar el marcador ahora si quel ora si que partiendo de las consideraciones teóricas del grupo Val. Es.Co. (Briz \& Pons, 2010; Briz, Pons \& Portolés, 2008; Pons, 2001) y del análisis del Corpus Sociolingüístico de la Ciudad de México (CSCM, Martín Butragueño \& Lastra, 2011, 2012, 2015). Para tal fin, en $\$ 2$ se presentan las características estructurales de ahora si quelora si que como marcador discursivo, en $\$ 3$ se describe la metodología empleada, en $\$ 4$ se lleva a cabo el análisis de las funciones, mientras que en $\$ 5$ se discuten los resultados y, finalmente, en $\$ 6$ se presentan las conclusiones.

\section{Sobre la habilitación de abora sí quelora sí que como marcador discursivo}

Los marcadores discursivos han sido descritos como:

unidades lingüísticas invariables, [que] no ejercen una función sintáctica en el marco de la predicación oracional y poseen un cometido coincidente en el discurso: el de guiar, de acuerdo con sus distintas propiedades morfosintácticas, semánticas y pragmáticas, las inferencias que se realizan en la comunicación. (Portolés, 1998, p. 27) 
Por sus funciones, el grupo Val.Es.Co. (Pons, 2001) los ha clasificado en: (i) conectores (argumentativos y metadiscursivos), que sirven para que el hablante articule sus estrategias argumentativas, organice el discurso y realice tareas de edición, como la formulación y la reformulación; (ii) modalizadores, que permiten dirigir la atención hacia una unidad discursiva, expresar un acuerdo, falso o genuino, y señalar la actitud del hablante con respecto al enunciado y la forma en la que se enfrenta al mensaje (Briz, 2008a; Pons, 2000), y (iii) controladores de contacto, que ayudan a gestionar la interacción, interpelando al interlocutor sea para verificar el acuerdo o monitorear la atención, por lo que se relacionan con aspectos de alteridad.

De acuerdo con Pons (1998), existen varios rasgos estructurales que permiten caracterizar a estos elementos discursivos. Fonológicamente se sitúan entre pausas, tienen su propia curva entonativa y tienden a presentar una reducción fónica, como en ora por ahora. Morfológicamente pueden estar compuestos por una o varias palabras, pero no pueden sufrir flexión, declinación o procesos de afijación, ni pueden ser modificados por otros elementos. Sintácticamente no son constituyentes de la oración ya que pueden ser elididos y esto no afecta ni a la estructura sintáctica ni a la gramaticalidad de la oración. Semánticamente carecen de significado léxico o, si lo tienen, es reducido, por lo que no contribuyen al contenido proposicional de la oración.

Pragmáticamente, contextualizan el enunciado lingüística y enunciativamente, enlazan el enunciado en el que aparecen con el contexto previo o con el subsecuente. Son marcas asociadas con la planificación discursiva que indican la forma en que el hablante cree que un enunciado se relaciona con otro. Por último, distribucionalmente son unidades polifuncionales que pueden establecer relaciones en diferentes niveles del discurso, también pueden combinarse con conjunciones y/o entre sí, pero no pueden constituir por sí mismos un enunciado. Muchos de ellos no se escriben porque pertenecen exclusivamente al ámbito de la lengua oral coloquial, como ahora sí quelora sí que. Así pues, por todas estas características, los marcadores discursivos son una clase funcional más que formal.

Considérense los siguientes ejemplos:

(4a) 1017 I: "ya es tiempo de que tu marido se decida a buscar casa/ ya vivieron allá/ ¡once años!”

$1018 \mathrm{E}: \mathrm{mm}$

1019 I: decía < - ecía> yo/ “ionce años! viviendo allá/ oye ahora < ora> si que di hija/ di casa/ y di todo"/ y estaban acabando con el departamento/ [jtodo!] (Entrevista 92).

(4b) 1019 I: decía < - ecía> yo/ “¡once años! viviendo allá/ oye di hija/ di casa/ y di todo”/ y estaban acabando con el departamento/ [itodo!] 
En (4b), se observa que el marcador puede ser suprimido y no se altera ni la estructura sintáctica ni el contenido proposicional de la unidad en la que aparece; esto atestigua su carácter parentético y su valor funcional como marcador discursivo.

(5) [hablando sobre las armas que manejan los policías]

534 I: y si estás en servicios de bancos// es este/ el M P < - eme pe> cinco/ o el M <- eme>/ el $\mathrm{M}<$ - eme> uno// o la mendoza/ que le dicen mendoza/ nueve milímetros/ es cuando estás en bancos/ cuando andas de custodio traes tu revólver treinta y ocho especial/ y una escopeta doce

$535 \mathrm{E}:$ esa/// que es/ ah/ sí sí

536 I: ahora <- ora si que es la...

537 E: ¡es una cortita así?

538 I: la mentada chaquetera (Entrevista 41).

En (5) se comprueba de nuevo que el marcador es opcional, ya que si se elide no se alteran los rasgos sintácticos ni semánticos de la unidad discursiva en la que aparece. Sin embargo, si contrastamos (4a) y (5) podemos notar que la función del marcador no es la misma, ya que en (5) desarrolla valores de reformulación, pues permite explicar una escopeta doce introduciendo la mentada chaquetera, y esto no sucede en (4a). Ahondaremos en esto más adelante.

\section{Metodología}

Para nuestro estudio se decidió utilizar el CSCM (Martín Butragueño \& Lastra, 2011, 2012, 2015) porque el estilo empleado en las entrevistas oscila entre dos polos: (i) la entrevista semi-informal — caracterizada por la tendencia a emplear la variante formaly (ii) la conversación grabada, que llega a mostrar pocas diferencias con respecto a la conversación coloquial entre conocidos. Así, según los autores, resultó común que las entrevistas comenzaran presentando una estructura semi-informal, pero, conforme se iban desarrollando, terminaran en conversaciones.

El CSCM consta de 108 entrevistas divididas en tres grupos de acuerdo con el nivel de instrucción: alto (36), medio (36) y bajo (36). ${ }^{2}$ Estos, a su vez, están divididos en tres grupos etarios: jóvenes (20-34), adultos (35-54) y mayores (55 en adelante). Por último, los informantes están agrupados por género. En cuanto a los temas de las entrevistas, son

2. El nivel bajo incluye a personas analfabetas, con primaria trunca o terminada, por lo que recibieron hasta 6 años de escolarización. El nivel medio está compuesto por personas que tuvieron hasta 12 años de escolaridad. Finalmente, el nivel alto incluye a hablantes que recibieron, al menos, 16 años de escolaridad. 
el trabajo, la familia y el tiempo libre, por lo que los tópicos fueron, hasta cierto punto, similares en todos los informantes.

Como punto de partida, se rastrearon manualmente todas las ocurrencias de ahora sí que y su forma reducida ora si que, aunque también se presentaron las formas aoa si que y oa si que. Esta búsqueda se realizó prestando atención al contexto discursivo en el que aparecían estas formas, fundamentalmente para asegurarnos de que se trataba de usos discursivos como los descritos en $\$ 2$. En total, el marcador apareció en 266 ocasiones —a lo largo de 53 entrevistas - distribuidas de la siguiente manera: ahora si que, 32 veces; ora si que, 231; oa sí que, 2 y aoa sí que, 1 vez. Como refleja la Tabla 1, los hombres, el nivel de instrucción medio y el grupo etario de jóvenes favorecen su empleo; en contraste, las mujeres, el nivel de instrucción alto y el grupo de edad de 55 años en adelante evitan su empleo. Estos datos ya han sido reportados por Aldama y Reig (2016).

TABla I. Distribución de las Variantes Del MarCador EN EL CORPUS

\begin{tabular}{|l|c|c|c|c|c|c|c|c|}
\hline & \multicolumn{3}{|c|}{ Nivel de instrucción } & \multicolumn{4}{c|}{ Edad } & \multicolumn{2}{c|}{ Género } \\
\hline & Alto & Medio & Bajo & Jóvenes & Adultos & Mayores & H & M \\
\hline $\begin{array}{l}\text { ahora } \\
\text { si que }\end{array}$ & 6 & 16 & 10 & 12 & 11 & 9 & 18 & 14 \\
\hline ora si que & 38 & 107 & 86 & 137 & 65 & 29 & 158 & 73 \\
\hline oa si que & & 1 & 1 & 2 & & & 1 & 1 \\
\hline aoa si que & & & 1 & 1 & & & & 1 \\
\hline
\end{tabular}

En cuanto a la variación de ahora sí que y ora sí que, el nivel medio es el que más emplea la forma reducida ( 107 veces), mientras que el alto es el que menos la usa (38 veces) y lo mismo sucedió con la forma normativa: apareció en 16 ocasiones en el nivel medio y 6 veces en el nivel alto. Por su parte, oa sí que ocurrió en el nivel medio (1) y en el nivel bajo (1), en tanto que aoa si que solo apareció en el nivel bajo. En este sentido, cabe mencionar que esta variación no obedece a una distinción funcional, ya que las dos formas más comunes pueden ser empleadas por un mismo hablante y, además, pueden aparecer en los mismos contextos, desempeñando las mismas funciones. En cuanto a las otras dos formas, su incidencia es realmente baja, por lo que tampoco puede considerarse que supongan distinciones funcionales. En consecuencia, el uso alternado de ahora si que y ora si que refleja la oposición entre las variantes normativa y coloquial. Más aún, la preferencia de la forma reducida ora si que no sería extraña, ya que en México es un fenómeno común la aféresis de ora por ahora. 


\section{Sobre las funciones de ahora síquelora síque}

Por la presencia del adverbio demostrativo ahora, el marcador conserva un matiz temporal —o de anclaje deíctico, si se prefiere— que influye en la interpretación de sus funciones. De tal forma, ahora si quelora si que tiene un valor (proto)típico que indica una pertinencia en el momento actual de la enunciación, esto es, el hablante se percata de que una unidad discursiva viene a propósito porque: (i) tiene relación con lo que se está hablando o (ii) porque sirve como una ejemplificación o ilustración.

(6) [hablando sobre idiomas]

334 I: mi mamá tenía que haber aprendido primero el chino/ y estaba medio

335 E: en chino

336 I: ahora < ora> si que en chino [(risa)]

337 E: [(risa)]

338 I: sí ahora si que en chino (Entrevista 69).

En el ejemplo (6), el marcador sirve para introducir una unidad discursiva —en chino- que viene ad hoc con la unidad que lo antecede y con el tema de la conversación: tenia que haber aprendido primero el chino y estaba medio. Esta coincidencia también es identificada por E en 335 .

(7) [hablando sobre su separación matrimonial]

84 I: pero ya había metido la pata de salirme de trabajar/ y jay en la torre!/ pues ya/ ni modo/ me quedé/ ahora si que como el perro de las/ [tort-/ de las dos tortas] (Entrevista 69).

En (7), en cambio, lo que introduce el marcador es una ejemplificación de la situación que está narrando I, y tal ejemplo es una frase hecha: el perro de las dos tortas, es decir, se quedó sin trabajo y sin marido. En este sentido, en varias ocasiones los hablantes recurren a este tipo de frases para intentar ser más claros:

(8) 130 I: pues <-pus> aquí nos tocó vivir/ ahora <-ora si que a la tierra que fueres [haz lo que vieres] (Entrevista 40).

(9) 135 E: oye pero/ ¿qui-/ ¿te enseñó alguien a dibujar o tú...?

136 I: no [el dibujo]

$137 \mathrm{E}:$ [tú solo] 
138 I: ya este <- este:> pues < pus> ahora < ora > si que ya lo traigo en la sangre ¿̨no? (Entrevista 75).

Así pues, el valor central del marcador podría ser parafraseado como "aquí sí aplica $\mathrm{X}$ ", asociado con dos funciones: una en la que se destaca la pertinencia o coincidencia de una unidad discursiva, como en (6), y otra que sirve para ejemplificar (Cuevas, 2020, comunicación personal), como en los casos de (7) a (9). Esta función fue la más común, ya que apareció en 127 ocasiones como marca de pertinencia y 30 veces como ejemplificador, 13 de ellas introduciendo una frase hecha. ${ }^{3}$

Ahora bien, como se comentó a propósito del ejemplo (5), el marcador también desempeña una función de reformulación, a través de la cual el hablante intenta expresar de mejor manera su idea para hacerla más relevante para el interlocutor (Cuenca y Bach, 2007).

(10) 260 I: pero lo que sí es que este/ de de entre otras gentes que han asistido/ a ese tipo de eventos/ por lo regular este/ encuentras libros/videos// pues/ ahora < ora> si que datos que te puedan aportar cómo van este/ qué han hecho// existen revistas/ que se mueven a nivel mundial (Entrevista 19).

(11) 659 I: “[o sea]/ yo creo que merecemos un poco de respeto y punto/ ¿no?/ yo traté en buen plan/ hacer plática y tú no quisiste/ te reístes (sic) de mí/ no va por ahí/ ¿sí??"// entonces <-entós> ya se fue/ se fue/ entonces <-entós> empecé a decirle a A/ “¿qué onda/ qué pasó?"/ "oye/ es que esto que...”/ "de acuerdo/ estoy <- toy> contigo/ discúlpame"/ ya/ ya traían pocas de copas/ ahora < ora> si que una

$660 \mathrm{E}:$ sí/ lo vi bien jarra

661 I: jarrísima (Entrevista 6).

(12) 550 I: mi papá es policía/ pero él está en otro lado (risa)/ ahora sí que él es el auxiliar/ yo soy de la bancaria (Entrevista 41).

En (10), el marcador introduce una reformulación, cuando el hablante engloba libros y videos en datos. En tanto que en (11), el informante reformula pocas de copas por una jarrísima — una forma coloquial de decir que se ha consumido alcohol en exceso- con

3. En el corpus hay 7 casos donde no hay contexto suficiente para determinar la función del marcador, principalmente porque el hablante no concluye su intervención, sea porque la deja suspendida o porque le arrebatan el turno. Así, estos 7 casos no fueron incluidos en los totales de cada función ni en el del valor secundario de formulación. 
lo que redimensiona la cantidad de alcohol ingerido. Finalmente, en (12), a través del marcador, el hablante introduce una explicación sobre por qué su papá está en otro lado: pertenecen a distintas corporaciones policiacas. Así pues, como reformulador, la función del marcador puede ser parafraseada como "aquí sí aplica mejor X que Y". Esta función se presentó en 87 ocasiones.

Ahora bien, un valor secundario asociado con este marcador es la formulación, que se ha descrito como una estrategia que le permite al hablante solventar los problemas propios de la planeación y la construcción del turno conversacional. En consecuencia, a los formuladores:

se les ha asignado un papel retardatorio; fórmulas, así pues, que permiten ganar tiempo para pensar y "planificar" lo que se va a decir a continuación; pausas para pensar, elementos que rellenan espacios vacíos cuando el hablante inicia su discurso, cuando no encuentra el modo de continuar. (Briz, 1993, p. 45)

Por su función, se presentan en contextos donde hay alargamientos vocálicos o consonánticos, falsos inicios, palabras cortadas, pausas y/o repeticiones:

(13) [hablando sobre cómo elaborar mole]

173 I: sí/ sale la pasta/ se < se:>/ se < se:> este/ para que no se haga bolas pues se/ s-/ ahora < ora $>$ si que se/ este/ se vacía en una <- una:> cazuela y (Entrevista 105).

(14) [hablando sobre el trabajo de I en una empresa]

663 I: "oiga pero es que yo/ yo lo desconozco"/ "no no/ tú/ te encargas de ahí"/ "ah caray/ bueno pues <- pus>”/ y sí me mandaron al < a:l>/ comedor de ahí

$664 \mathrm{E}: \mathrm{mm}$

665 I: el/ el comedor/// e- e- era el que < que::>/ ¿cómo decirte?/ era <- era::>/ ahora $<$ ora si que donde iba a comer todo mundo/ ¿no? (Entrevista 62).

En (13), I tiene dificultades para seguir con su explicación y a través del marcador, junto con las repeticiones, alargamientos vocálicos y falsos inicios, retarda su intervención hasta que logra concluirla. Algo similar sucede en (14): la informante tiene problemas para reformular, lo que se ve reflejado en la presencia de palabras cortadas, repeticiones, alargamientos vocálicos y la frase ¿cómo decirte?. En este sentido, hay casos donde los hablantes no consiguen ejemplificar o reformular, por lo que el marcador contribuye a la formulación: 
(15) [hablando sobre un altercado entre locatarios de un mercado]

1278 I: [y] aun así/ pero pues < ps> es que después/ ¡ella amenazó a los!/ a los demás dirigentes/ que ya no les iban a dar lugares <-lusgares>

$1279 \mathrm{E}: \mathrm{mh}$

1280 I: que le les/ ahora < ora> sí que ¡los iban a este! ¡cómo dijo?// ¡como cuando < cuando:>!/ entra uno a la cárcel y los/ los este/ ¡cómo le dicen? (Entrevista 83).

Así, en (15), I sufre un olvido y no logra concluir su ejemplificación/reformulación, y dicha incapacidad se extiende a lo largo de su intervención. Ahora bien, es importante señalar que el marcador - junto con las pausas, los alargamientos, las repeticiones, etcétera- es parte de la estrategia de edición que lleva a cabo el hablante, una en la que se tiene que ganar tiempo para poder pensar y continuar con la intervención. Finalmente, este valor secundario ocurrió 15 veces.

Por último, queremos reiterar que el marcador viene acompañado de un matiz modal cuando cumple con las funciones de "aquí sí aplica X” y "aquí sí aplica mejor X que Y”. En el primer caso, el hablante se percata de la pertinencia de una unidad discursiva y la reviste con una mayor prominencia comunicativa, como en el caso de (16), donde se destaca la presencia de Tacubaya a través de varios recursos: el marcador, la exclamación y el controlador de contacto ¿eh?:

(16) [desde el inicio de la entrevista I mencionó que nació en la colonia Tacubaya] 102 I: y ya me/ me fui a aprender la fotografía con un compadre de mi papá una fotografía muy famosa en Tacu-l ahora si que en ¡Tacubaya! ¿eh? (Entrevista 98).

En el caso de la reformulación, ya se ha explicado que dicho proceso ayuda al hablante a comunicarse de manera más precisa, por lo que la unidad discursiva que introduce el reformulador es la que ha de tomarse en cuenta para que la comunicación prosiga. De tal forma, el hablante señala ostensivamente que hay partes del discurso que son comunicativamente más prominentes.

En resumen, se han descrito tres funciones del marcador: (i) una que señala la pertinencia o coincidencia de una unidad discursiva en un momento particular de la enunciación, (ii) otra que ejemplifica o ilustra y (iii) una más que reformula. Además, se señaló que hay dos valores secundarios que acompañan al marcador: la formulación y la modalización.

\section{Discusión}

De acuerdo con el análisis, se identificaron dos funciones generales que pueden ser parafraseadas como: (i) “aquí sí aplica X” y (ii) “aquí sí aplica mejor X que Y”. La primera 
incluye dos funciones más, una que indica la pertinencia de una unidad discursiva en el momento actual de la enunciación y otra que ejemplifica o ilustra lo que se está diciendo. En cuanto a la segunda función general, corresponde a la reformulación, por medio de la cual el hablante intenta expresar mejor sus ideas. Justo esta es la función que ha sido descrita por Aldama y Reig (2016). Creemos que estas dos funciones generales han quedado bien atestiguadas en nuestros datos, por lo que no nos detendremos a mencionar nada más.

Por otro lado, también se identificaron dos valores secundarios que pueden acompañar a estas funciones de ahora si quelora si que, específicamente nos referimos a la modalización y a la formulación. En el primer caso, el marcador viene acompañado de un matiz modal cuando el hablante ejemplifica o reformula, ya que trata de ser más claro y, por tanto, más relevante para lograr que el interlocutor comprenda mejor lo que se le está diciendo. De tal forma, la reformulación, por ejemplo," [it] is not only reworded in a different way, but it is elaborated in a better, more relevant way, at least from the speaker's perspective" (Cuenca \& Bach, 2007, p. 152; énfasis en el original). En consecuencia, las funciones del marcador se encuentran asociadas con rasgos de cooperación (Grice, 1975) y relevancia (Sperber $\&$ Wilson, 1986), ya que el hablante indica que hay partes del discurso que son más importantes que otras y que son sobre las que hay que poner mayor atención. Esto mismo sucede cuando el marcador se emplea para señalar la pertinencia de un elemento discursivo en un punto particular de la intervención; nos referimos a casos como (6) y (16), donde ahora sí quelora sí que actúa como una marca lingüística de relevancia que contribuye a destacar una unidad discursiva particular (Guillén, 2018). Finalmente, este valor secundario de modalización está asociado con el anclaje deíctico que conserva el marcador y que señala un momento particular de la enunciación, uno donde la introducción de una unidad discursiva resulta pertinente.

En cuanto al valor secundario de formulación, se explicó que el marcador es parte de la estrategia de edición discursiva del hablante, esto es, su empleo lo auxilia en la construcción de su intervención, pues le permite ganar tiempo mientras encuentra la manera de seguir adelante. En varios casos, esto sucedió cuando el hablante no lograba concretar la ejemplificación o la reformulación y, en consecuencia, aparecen titubeos, repeticiones o falsos inicios, que atestiguan el fracaso del hablante por cumplir tales funciones, y el marcador se emplea para retrasar la intervención. En este sentido, se ha descrito que otros reformuladores también desempeñan esta labor, por ejemplo, Briz (2008b) ha señalado que $o$ sea "en el habla puede aparecer a veces con el valor de expletivo o muletilla, a modo de pausa léxica para pensar lo que sigue a continuación", y esto mismo parece ocurrir en algunos casos con ahora sí quelora sí que. Por último, hay que señalar que modalización y formulación son dos valores secundarios que parecen excluirse, esto es, en la formulación no hay un valor modal en el marcador por el simple hecho de que no hay una ejemplificación, ilustración o reformulación; solo hay un intento por retrasar la comunicación, mientras se halla la forma de continuar.

En breve, las funciones generales de ahora si quelora si que pueden ser agrupadas en la categoría de conectores metadiscursivos propuesta por el grupo Val.Es.Co. (Pons, 2001), 
es decir, aquellos que están relacionados con la organización de la actividad discursiva y con la técnica que emplean los hablantes para formular y producir sus mensajes (Briz, 1993). En este sentido, el valor secundario de formulación también pertenece a esta categoría; mientras que el valor secundario de modalización, como resulta natural, está relacionado con los modalizadores, marcadores que indican la prominencia comunicativa de ciertas parcelas discursivas.

\section{Conclusiones}

Nuestro objetivo era caracterizar las funciones pragmático-discursivas que ha desarrollado el marcador ahora sí quelora sí que. Para empezar, se estableció que ahora sí que y ahora síno son formas análogas, por lo que nos centramos únicamente en el primer marcador. Enseguida, se distinguió que, por su origen, el marcador conserva un rastro de anclaje deíctico que se asocia con la noción de pertinencia en el punto actual de la conversación, y, a partir de esto, el marcador ha desarrollado las funciones de: (i) marca de pertinencia, (ii) ejemplificador y (iii) reformulador, donde las dos primeras pueden ser parafraseadas como "aquí sí aplica X" y la última como "aquí sí aplica mejor X que Y".

También se describieron dos valores secundarios que pueden acompañar al marcador: la formulación y la modalización. En el primer caso, se presenta cuando el hablante fracasa en sus intentos por ejemplificar o reformular, lo que promueve la aparición de otras marcas - como pausas, alargamientos, etcétera — que atestiguan estas dificultades. Con este valor, el marcador se emplea para ganar tiempo y pensar cómo continuar, aunque a veces no se consiga, como en el caso de (15). En cuanto a la modalización, se encontró que está presente en todas las funciones del marcador, excepto cuando tiene el valor secundario de formulador.

En resumen, además de la función de reformulación, propuesta por Aldama y Reig (2016), aquí agregamos dos más: (i) marca de pertinencia y (ii) ejemplificación, y proponemos dos valores secundarios: (a) formulación y (b) modalización. Entonces, pertinencia, ejemplificación, reformulación y formulación están relacionados con los marcadores metadiscursivos, mientras que los modalizadores lo están con la modalización.

Finalmente, cabe mencionar que este es un primer intento por detallar las funciones del marcador discursivo ahora si quelora sí que, por lo que serán necesarios más estudios que abonen la descripción de este marcador, tan característico de la variante del español de México.

\section{Referencias}

Aldama, J. D., \& Reig, A. (2016). Variación sociolingüística en el empleo de un nuevo marcador discursivo: ahora si que en el español de México. Boletín de Filología, 52(2), $15-47$.

Briz, A. (1993). Los conectores pragmáticos en español coloquial (II): su papel metadiscursivo. Español Actual, 59, 39-56. 
Briz, A. (2008a). Presentación. En Diccionario de partículas discursivas del español. Recuperado de http://www.dpde.es/\#/intro/code_page_sect_0.

Briz, A. (2008b). O sea. En Diccionario de partículas discursivas del español. Recuperado de http://www.dpde.es/\#/entry/osea2.

Briz, A., \& Pons, S. (2010). Unidades, marcadores y posición. En Ó. Loureda y E. Acín (Coords.), Los estudios sobre marcadores del discurso, hoy (pp. 523-557). Madrid: Arco.

Briz, A., Pons, S., \& Portolés, J. (Coords.) (2008). Diccionario de partículas discursivas del español. Recuperado de http://www.dpde.es.

Cuenca, M. J., y Bach, C. (2007). Contrasting the form and use of reformulation markers. Discourse Studies, 9(2), 149-174.

Cuevas, V. (20 de enero de 2020). Comunicación personal.

Grice, H. P. (1975). Logic and conversation. En P. Cole \& J. Morgan (Eds.), Syntax and semantics 3: Speech acts (pp. 41-58). Nueva York: Academic Press.

Guillén, J. E. (2018). Hacia una caracterización funcional de fijarse como marcador del discurso. Pragmalingüistica, 26, 131-147. doi: http://dx.doi.org/10.25267/ pragmalinguistica.2018. I26.07.

Martín Butragueńo, P., y Lastra, Y. (Coords.) (2011). Corpus sociolingüistico de la ciudad de México. Vol. 1: Hablantes de instrucción alta. México: El Colegio de México. Recuperado de http://lef.colmex.mx/index.php/investigaciones/corpussociolingueistico-de-la-ciudad-de-mexico-cscm.

Martín Butragueño, P., \& Lastra, Y. (Coords.) (2012). Corpus sociolingüistico de la ciudad de México. Vol. 2: Hablantes de instrucción media. México: El Colegio de México. Recuperado de http://lef.colmex.mx/index.php/investigaciones/corpussociolingueistico-de-la-ciudad-de-mexico-cscm.

Martín Butragueño, P., \& Lastra, Y. (Coords.) (2015). Corpus sociolingüistico de la ciudad de México. Vol. 3: Hablantes de instrucción baja. México: El Colegio de México. Recuperado de http://lef.colmex.mx/index.php/investigaciones/corpussociolingueistico-de-la-ciudad-de-mexico-cscm.

Pons, S. (1998). Conexión y conectores. Estudio de su relación en el registro informal de la lengua. Valencia: Quaderns de Filologia.

Pons, S. (2000). Los conectores. En A. Briz (Ed.), ¿Cómo se comenta un texto coloquial? (pp. 193-220). Barcelona: Ariel.

Pons, S. (2001). Connectives/discourse markers. An overview. En H. Ferrer y S. Pons (Eds.), La pragmática de los conectores y las partículas modales (pp. 219-243). Valencia: Quaderns de Filologia.

Portolés, J. (1998). Marcadores del discurso. Barcelona: Ariel.

PRESEEA. Proyecto para el estudio sociolingüístico del español de España y de América (2008). Marcas y etiquetas minimas obligatorias. Recuperado de http://www.linguas. net/preseea.

Sperber, D., \& Wilson, D. (1986). Relevance: Communication and Cognition. Oxford: Basil Blackwell. 


\section{Apéndice: Marcas y etiquetas mínimas obligatorias (tomado de PRESEEA, 2008, p. 7).}

\begin{tabular}{|l|l|}
\hline ORTOGRAFÍA Y PUNTUACIÓN & \\
\hline i! & Enunciados exclamativos \\
\hline ¿? & Enunciados interrogativos \\
\hline I & Pausa mínima \\
\hline /I & Pausa \\
\hline$:$ & Tras código de hablante (I: E: A1: ) \\
\hline Mayúsculas & Inicial de nombres propios y siglas \\
\hline Elementos cuasi-léxicos funcionales & $\begin{array}{l}\text { Interjecciones; apoyos. } \\
\text { Escritura ortográfica (ah, ay, aha, mmm, eeh, pff, bah) }\end{array}$ \\
\hline Onomatopeyas & Escritura ortográfica (zas, bum, plas) \\
\hline
\end{tabular}

\begin{tabular}{|l|l|}
\hline ETIQUETADO DE RUIDOS & \\
\hline$<$ ruido = " "/> & $\begin{array}{l}\text { Ruido, con especificación de tipo (p.e. <ruido = } \\
\text { "chasquido boca"/>) I }\end{array}$ \\
\hline$<$ ruido_fondo></ruido_fondo> & Ruido continuo de fondo AD \\
\hline$<$ risas = " "/> & $\begin{array}{l}\text { Risas, con especificación de emisor/es (p.e. <risas = } \\
\text { "E"/>, <risas = "todos"/>) I }\end{array}$ \\
\hline$<$ <entre_risas></entre_risas> & Risas simultáneas con el habla AD \\
\hline$<$ registro_defectuoso></registro_defectuoso> & Fragmento de la grabación de mala calidad AD \\
\hline <interrupción_de_grabación/> & Interrupción de la grabación I \\
\hline
\end{tabular}

\begin{tabular}{|l|l|}
\hline ETIQUETADO FÓNICO & \\
\hline <énfasis></énfasis> & Fragmento con pronunciación claramente enfática AD \\
\hline <alargamiento/> & Alargamiento de sonido D (sin espacios) \\
\hline$<$ silencio/> & Silencio de un segundo o más I \\
\hline$<$ palabra_cortada/> & Palabra cortada D \\
\hline <vacilación/> & Vacilación; titubeo breve I \\
\hline$<$ sic></sic> & No es descuido de transcripción AD \\
\hline$<$ ininteligible/> & Fragmento ininteligible I \\
\hline
\end{tabular}

\begin{tabular}{|l|l|}
\hline ETIQUETADO LÉXICO & \\
\hline$\langle$ término $></$ término $>$ & Lexía claramente usada como uso especializado AD \\
\hline$\langle$ extranjero $></$ extranjero $>$ & Extranjerismo (excepto usos de la L2 del hablante) AD \\
\hline$\langle$ siglas $=[]></$ siglas $>$ & Siglas; incluye pronunciación $A D$ \\
\hline
\end{tabular}

\begin{tabular}{|l|l|}
\hline ETIQUETADO DE DINÁMICA DISCURSIVA & \\
\hline$<$ cita $<</$ cita $>$ & Cita, estilo directo AD \\
\hline$<$ simultáneo $></$ simultáneo $>$ & $\begin{array}{l}\text { Solapamiento (traslape). También se usa en turnos de } \\
\text { apoyo, si fuera necesario AD }\end{array}$ \\
\hline
\end{tabular}

\begin{tabular}{|l|l|}
\hline ETIQUETADO DE LENGUA & \\
\hline$<$ lengua $="$ " $></$ lengua $>$ & $\begin{array}{l}\text { Cambio de lengua (léxico, oracional, } . . .) \text {, especialmente } \\
\text { L2 del hablante, con indicación desarrollada de lengua } \\
\text { (p.e. }<\text { lengua }=\text { "gallego" }></ \text { lengua }>A D\end{array}$ \\
\hline
\end{tabular}

\begin{tabular}{|l|l|}
\hline ETIQUETADO DE TRANSCRIPCIÓN & \\
\hline$<$ transcripción_dudosa ></transcripción_dudosa> & Transcripción dudosa para transcriptor y revisores AD \\
\hline$<$ tiempo $="$ "/> & $\begin{array}{l}\text { Anotación de minuto y segundo de grabación. (p.e. } \\
<\text { tiempo }=" 02: 45 " />\text { ) I }\end{array}$ \\
\hline$<$ <observación_complementaria = " "/> & Observación complementaria I \\
\hline
\end{tabular}

